\title{
Estimation of Reference Values for PFOS and PFOA in Human Biomonitoring and Relevance of Exposure among Family Members in China
}

\author{
Wei Liu', Guanghui Dong ${ }^{2}$, Yingwei Luo ${ }^{3}$, Li Liu ${ }^{3}$ Ziwei $\mathrm{Cao}^{1}, \mathrm{Xiana} \mathrm{Li}^{1}$, Yihe $\mathrm{Jin}^{1 *}$ \\ ${ }^{1}$ School of Environmental Science and Technology, Dalian University of Technology, Key Laboratory of Industrial Ecology and \\ Environmental Engineering, Ministry of Education, Dalian, China; ${ }^{2}$ School of Public Health, China Medical University, Shenyang, \\ China; ${ }^{3}$ The First Hospital of China Medical University, Shenyang, China. \\ Email: ${ }^{*}$ jinyihe@dlut.edu.cn
}

Received February 19 $9^{\text {th }}, 2012$; revised March 10 ${ }^{\text {th }}, 2012$; accepted April $6^{\text {th }}, 2012$

\begin{abstract}
The reference values of serum perfluorooctane sulfonate (PFOS) and perfluorooctanoate (PFOA) concentrations were evaluated based on the human blood samples collected from Kashi, Xinjiang. And human serum samples of family members from Liaoning were evaluated for levels of PFOS and PFOA with the purpose to compare exposure pathways for family members. Among the 110 blood specimens from Kashi, the detection frequency of PFOS and PFOA was $93 \%$ and $6 \%$, respectively. Reference values of serum PFOS, evaluated as the 90th percentiles of the concentrations, were determined to be $6.44 \mu \mathrm{g} / \mathrm{L}$. Significant positive correlations were observed for serum levels of PFOS and PFOA among family members in Liaoning. Specially, stronger correlation between mother and offspring was observed than that between father and offspring. And stronger correlation of serum PFOS and PFOA levels was observed among family members in rural areas than those in big and small-medium cities. Difference in the association of serum PFOS and PFOA level among family members suggested that exposure in the outdoor and working environment of different occupations should be evaluated. Present study provides reference values for exposure assessment in China and potential pathways of human exposure to PFOS and PFOA.
\end{abstract}

Keywords: Perfluorinated Compounds; Human Biomonitoring; Reference Value; Exposure; Family Member

\section{Introduction}

Increasing number of researches reported extensive human exposure to perfluorinated compounds (PFCs) in different geographical locations around the globe [1-3]. Perfluorooctane sulfonate (PFOS) and perfluorooctanoate (PFOA), two most widely known PFCs, are frequently detected at higher levels compared with other PFCs [4-6]. Some subpopulations, living in proximity to or working in fluorochemical manufacturing plants, were found with high body load of PFCs [7,8]. Into the far future humans will continue to receive a fairly constant, low level exposure to PFCs from legacy sources, although the fluorine industry has announced a commitment to the phaseout of eight carbon perfluoroalkyl chemistry [9]. An increase in human exposure to PFOS and PFOA over time was even observed in some area in Japan and China [10, 11]. Further, animal experiments and epidemiological studies found various toxicities of PFOS and PFOA, leading to increasing concern in health risk posed by

${ }^{*}$ Corresponding author. these pollutants [2,12-15].

Elucidation of PFCs sources is crucial for prevention and control of human exposure. However, exposure pathways to PFCs prove to be complicated and remain not well known, because humans are exposed to PFCs via a vast number of pathways over an extended period of time. Possible pathways of human exposure to PFCs include inhalation of volatile and neutral PFCs, ingestion of non-volatiles attached to particles, and exposure of contaminated food and water [2,3]. Food intake is regarded to be the most important exposure pathway for the background population, whereas drinking water is dominant for populations near sources of contaminated drinking water [15]. Further research is warranted to elucidate the sources of human exposure to PFCs.

In the present study, the reference values of serum PFOS and PFOA concentrations were evaluated based on the human blood samples collected from Kashi, Xinjiang. In this remote region with extremely low density of population and industry, the inhabitants mainly live on farming and stock raising. Therefore, the area is expected 
to be posed to a light PFCs contamination. Our previous study has found extremely low PFOS and PFOA concentrations in the surface water and tap water in Xinjiang [16]. It is of particular interest to investigate the levels of human exposure to PFOS and PFOA in Kashi, in order to provide background data of human biomonitoring and serve for the exposure assessment in the general population. Further, human serum samples of family members from Liaoning, including the fathers, mothers, and offspring, were evaluated for levels of PFOS and PFOA with the purpose to compare exposure pathways for family members. Relatively higher PFOS and PFOA concentrations have been detected in human serum from Liaoning $[17,18]$. Higher-level similarity in the living environment and dietary reduce the variance in exposure and provide valuable information for exposure pathways to PFCs.

\section{Materials and Methods}

\subsection{Research Ethics Board Approval}

Blood sampling involved in the study protocol was reviewed and approved by the China Medical University Research Ethics Board. Informed consent was obtained from all participants and parents or guardians provided consent for participants $<18$ years of age.

\subsection{Study Population and Blood Sampling}

The blood sampling was performed in 2007. For the determination of reference values for serum PFOS and PFOA, 110 human blood samples (58 males and 52 females) were drawn from volunteers recruited among Uyghurs living in Kashi, Xinjiang Uyghur Autonomous Region. All volunteers were 11 to 86 years old. They live on farming and stock raising without occupational exposure to PFCs. Blood samples of family members were collected from 44 families in areas from Liaoning province representative of big cities (Shenyang and Anshan, 11 families), small-medium cities (Yingkou and Liaoyang, 15 families), and rural areas around Shenyang (18 families). The cities were ranked according to population, denoted as big cities with population larger than 2 millions, and small-medium cities with population less than 2 millions. The family members group consisted of subjects from different working environments expected to be non-occupationally exposed to PFCs. The blood samples of the father, mother, and one of the offsprings (son or daughter, random recruiting) were collected from each family. The ages of father, mother, son and daughter were in the range of $22-71,22-69,1-31$, and $1-35$ years old, respectively. Following the collection, the serum was separated from whole blood via centrifugation and stored at $-4^{\circ} \mathrm{C}$ prior to extraction and analysis.

\subsection{Reagents}

Heptadecafluorooctane sulfonic acid potassium salt and pentadecafluorooctanoic acid ammonium salt ( $>98 \%$ purity) were purchased from Fluka (Milwaukee, WI, USA) as standards for PFOS and PFOA, respectively. The internal standard $\left[1,2-{ }^{13} \mathrm{C}_{2}\right]$ perfluorooctanoate was donated by the Environmental Protection Agency of the USA ( $>99 \%$ purity, originally synthesized by Perkin Elmer, Boston, MA, USA). Tetrabutylammonium hydrogen sulfate (high-performance liquid chromatography [HPLC] grade) was obtained from Acros Organics (Geel, Belgium). Sodium carbonate ( $>99.5 \%$ purity) was obtained from Kanto Chemical (Tokyo, Japan). Nitrogen gas ( $>99.99 \%$ purity) was obtained from Japan Fine Products Corporation (Kawasaki, Japan). Methyl tertiary-butyl ether (MTBE; HPLC grade), methanol (HPLC grade), acetonitrile (HPLC grade) and ammonium acetate $(>97 \%$ purity) were obtained from Wako Pure Chemical Industries (Osaka, Japan). Deionized water was passed through a Presep-C Agri cartridge (Wako Pure Chemical Industries, Osaka, Japan) to remove any remaining contamination, called PFC-free water.

\subsection{Determination of PFOS and PFOA in Serum}

The internal standard $\left[1,2-{ }^{13} \mathrm{C}_{2}\right]$ perfluorooctanoate was added to each serum sample prior to extraction. The serum samples were extracted using a previously described method [19]. Briefly, $0.5 \mathrm{~mL}$ of serum, $1 \mathrm{~mL}$ of $0.5 \mathrm{M}$ tetrabutylammonium hydrogen sulfate solution and $2 \mathrm{~mL}$ of $0.25 \mathrm{M}$ sodium carbonate buffer were added to a 15$\mathrm{mL}$ polypropylene tube and mixed. Following addition of $5 \mathrm{~mL}$ of MTBE to the solution, the organic and aqueous layers were separated by centrifugation, and the organic layer was removed. The aqueous mixture was rinsed with MTBE and separated twice. The solvent was evaporated at room temperature under a nitrogen gas flow. The sample was then reconstituted in $0.5 \mathrm{~mL}$ of methanol. The sample was then passed through a nylon filter (Autovial R5 PUNYL; $0.45-\mu \mathrm{m}$ pore size; Whatman Japan, Tokyo) to remove any suspended materials and insoluble particles.

Each extracted solution was analyzed by liquid chromatography-mass spectrometry (LC/MS) as previously described [11]. Briefly, each methanol extract (injection volume, $10 \mu \mathrm{L}$ ) was chromatographed using HPLC (Agilent 1100 with Zorbax XDB C-18 [3.5 $\mu \mathrm{m}, 2.1 \times 150$ $\mathrm{mm}]$ ) at a flow rate of $0.2 \mathrm{~mL} / \mathrm{min}$. Gradient conditions were used in the mobile phase. Initial mobile phase conditions were 35:65 $\mathrm{CH}_{3} \mathrm{CN} / 10 \mathrm{mM} \mathrm{CH}_{3} \mathrm{COONH}_{4}$ buffer, followed by a $5 \mathrm{~min}$ ramp to $45: 55$, hold until $20 \mathrm{~min}$. 
The chromatographic column was kept at $40^{\circ} \mathrm{C}$. Mass spectra were taken using an LC/MS system equipped with an orthogonal spray interface (Agilent 1100MSD $\mathrm{SL}$ ), employing electrospray ionization in the negative mode. The selected ion monitoring was used for quantification of the analyses. The quantification ions were $\mathrm{m} / \mathrm{z}$ 413 (C7F15CO2-) for PFOA and m/z 499 (C8F17SO3-) for PFOS. The fragment of PFOA m/z 369 (C7F15-) and fragment of PFOS m/z 99 (FSO3-) were also monitored as qualifying ions.

\subsection{Quality Assurance/Quality Control}

Blanks using PFC-free water were handled with the same procedure as unknown samples. None of the blanks showed significant contamination. Calibration standards were analyzed every 10 samples. Matrix spike studies at concentration of $10 \mu \mathrm{g} / \mathrm{L}$ in serum were performed to evaluate the recovery. The mean extraction recoveries from the spiked serum samples ranged from $93 \%$ to $101 \%$ and from $98 \%$ to $109 \%$ for PFOS and PFOA, respectively ( $\mathrm{n}=10$ for each compound). The limit of detection (LOD) and limit of quantification (LOQ) were considered to be 3- and 10-fold larger than the signal-tonoise ratio, respectively. The LOD and LOQ were 0.01 $\mu \mathrm{g} / \mathrm{L}$ and $0.03 \mu \mathrm{g} / \mathrm{L}$, respectively, for both PFOS and PFOA.

\subsection{Statistics}

All statistical analyses were carried out using SPSS 13.0 (SPSS Inc., Chicago, IL). Values of $\mathrm{p}<0.05$ were considered to indicate statistical significance. All samples below the LOQ were treated as half the LOQ in the calculations. The serum levels of PFOS and PFOA were tested for their normal or log-normal distribution by the Kolmogorov-Smirnov test. And correlation coefficients were executed by Pearson test in data from a normal distribution and Spearman rank test in data with a nonnormal distribution. Wilcoxon matched pairs ranks test was used for comparison between various groups.

\section{Results and Discussions}

\subsection{Reference Values of PFOS and PFOA Concentrations in Human Serum}

Among the 110 blood specimens, the detection frequency of PFOS and PFOA in the samples was 93\% (102 of 110) and $6 \%$ (7 of 110), respectively. The PFOS and PFOA levels, stratified by sex, are summarized in Table 1. The median serum PFOS level was $1.92 \mu \mathrm{g} / \mathrm{L}$ in the range of $<0.03-22.6 \mu \mathrm{g} / \mathrm{L}$. Serum PFOA concentration was lower than PFOS with a median concentration of $<0.03 \mu \mathrm{g} / \mathrm{L}$. Reference values of serum PFOS, evaluated as the 90th
Table 1. Summary of serum PFOS and PFOA levels in human serum from Kashi, Xinjiang $(\mu \mathrm{g} / \mathrm{L})$.

\begin{tabular}{|c|c|c|c|c|}
\hline & & Male & Female & Total \\
\hline & $n$ & 58 & 52 & 110 \\
\hline \multirow[t]{4}{*}{ Age } & Mean \pm SD & $45.8 \pm 17.3$ & $40.6 \pm 17.5$ & $43.3 \pm 17.5$ \\
\hline & Mean \pm SD & $3.71 \pm 4.27$ & $2.06 \pm 2.62$ & $2.93 \pm 3.67$ \\
\hline & GM & 2.12 & 0.91 & 1.42 \\
\hline & Min & $<0.03$ & $<0.03$ & $<0.03$ \\
\hline \multirow[t]{7}{*}{ PFOS } & $25 \%$ percentile & 1.23 & 0.83 & 1.00 \\
\hline & Median & 2.39 & 1.20 & 1.92 \\
\hline & $75 \%$ percentile & 4.40 & 2.77 & 3.43 \\
\hline & Max & 22.6 & 17.2 & 22.6 \\
\hline & Mean $\pm \mathrm{SD}$ & $0.12 \pm 0.53$ & $0.21 \pm 1.08$ & $0.16 \pm 0.84$ \\
\hline & GM & $<0.03$ & $<0.03$ & $<0.03$ \\
\hline & Min & $<0.03$ & $<0.03$ & $<0.03$ \\
\hline \multirow[t]{4}{*}{ PFOA } & $25 \%$ percentile & $<0.03$ & $<0.03$ & $<0.03$ \\
\hline & Median & $<0.03$ & $<0.03$ & $<0.03$ \\
\hline & $75 \%$ percentile & $<0.03$ & $<0.03$ & $<0.03$ \\
\hline & Max & 3.50 & 7.70 & 7.70 \\
\hline
\end{tabular}

percentiles of the concentrations, were determined to be $6.44 \mu \mathrm{g} / \mathrm{L}$. And reference value of serum PFOA was not evaluated due to its low detection frequency.

Higher serum PFOS concentrations than PFOA has also been reported in China in other studies $[11,18]$. Serum PFOS concentrations in males were significantly higher than females, consistent with many other studies $[2,3]$. Comparisons of serum PFOS levels between different ages demonstrated a lack of significant age-related associations. Similar results have also been observed in China and other countries $[3,18]$.

Serum PFOS and PFOA concentrations in Kashi are much lower compared with the findings from other studies conducted in different countries and areas including China, as shown in Figure 1. Serum samples obtained in China during 2002 showed median PFOS and PFOA concentrations of $22.4 \mu \mathrm{g} / \mathrm{L}$ and $4.3 \mu \mathrm{g} / \mathrm{L}$ in 119 donors from Shenyang, $7.4 \mu \mathrm{g} / \mathrm{L}$ and $1.0 \mu \mathrm{g} / \mathrm{L}$ in 112 donors from Chongqing, respectively $[11,17]$. Also, Yeung et al. (2006) collected 85 samples of whole human blood from nine cities (eight provinces) in China, and the mean concentration of PFOS was highest in the samples collected from Shenyang $(79.2 \mu \mathrm{g} / \mathrm{L})$ and lowest in samples from Jintan $(3.72 \mu \mathrm{g} / \mathrm{L})$.

Compared with other regions of China, agriculture remains the main economic activity in Xinjiang Uyghur 


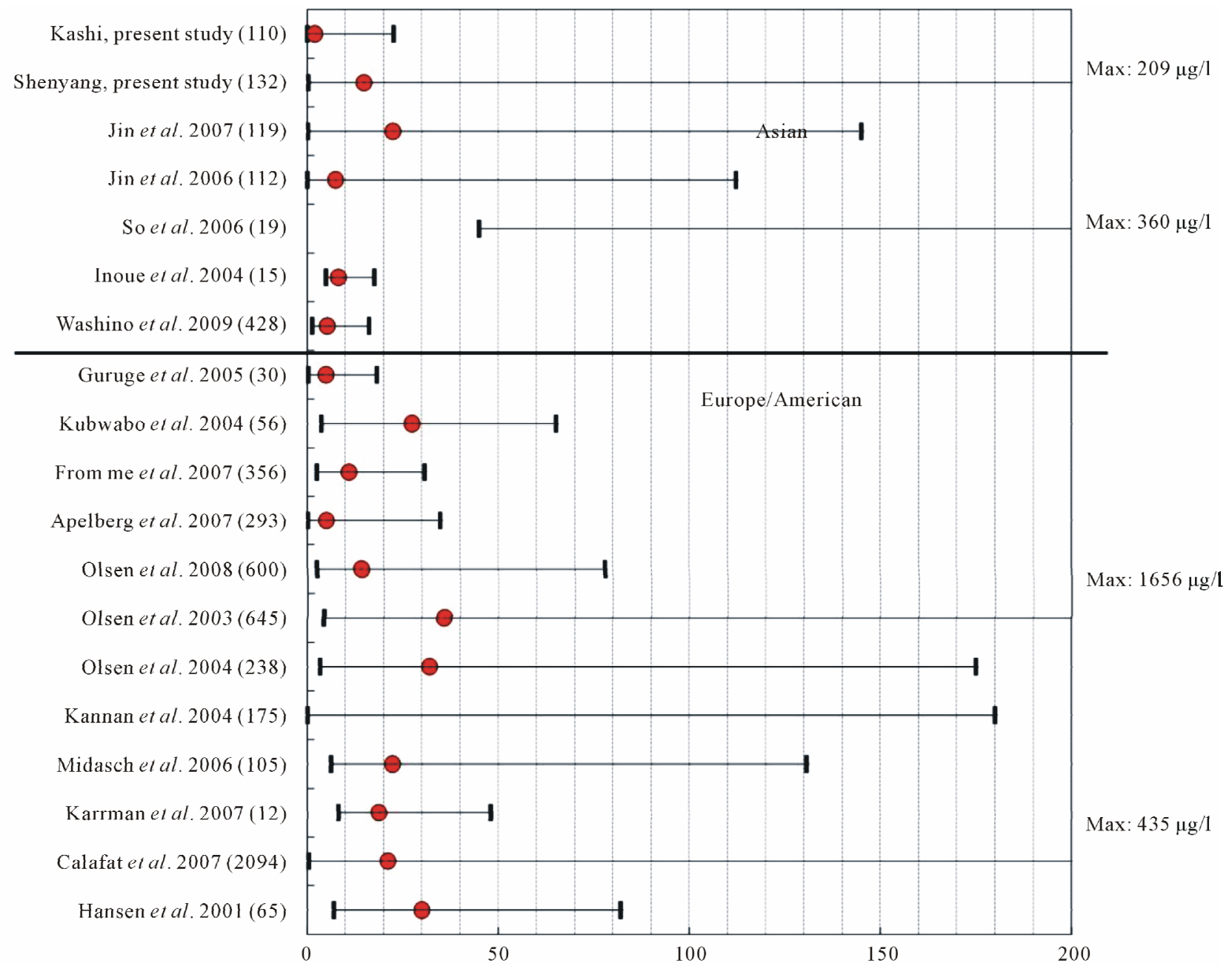

Figure 1. Comparison between the present study and the referential values of sample number (in brackets), minimum, median and maximum values of PFOS in human blood samples.

Autonomous Region. Due to the relatively less development in the economy and lower population and industry density, this remote region is possibly posed to a relatively light PFCs contamination. In the tap water collected from Atushi and Urumuqi in Xinjiang in 2006, PFOS and PFOA were both not detectable [16]. Thus, the lower serum levels of PFOS and PFOA in Xinjiang may result from the less sources of the PFCs exposure. And the serum PFOS and PFOA levels in Xinjiang provide an appropriate reference values for exposure assessment in China.

\subsection{Biomonitoring Values of Family Members}

Serum PFOS and PFOA levels of family members from Liaoning were summarized in Table 2. The serum PFOS and PFOA levels of the family members in Liaoning were in the range of $0.31-209 \mu \mathrm{g} / \mathrm{L}$ and $0.18-39.8 \mu \mathrm{g} / \mathrm{L}$, respectively, similar to the biomonitoring values in Shen- yang in 2002, where PFOS and PFOA were detected in the range of $0.24-145 \mu \mathrm{g} / \mathrm{L}$ and $0.24-59.8 \mu \mathrm{g} / \mathrm{L}$, respectively $[11,17]$ observed higher PFOS concentrations (range: 31.7 - $310 \mu \mathrm{g} / \mathrm{L}$ ) than the present study in Shenyang in 2004 and lower PFOA concentrations (range: 0.5 - $2.4 \mu \mathrm{g} / \mathrm{L}$ ). Human serum concentrations of PFOS and PFOA in Liaoning were obviously higher than the values in Kashi. The median serum PFOS concentrations in fathers, mothers, sons, and daughters were 4.1, 2.3, 1.9, and 1.0 times higher than the reference value.

Paired comparison between father and mother from the same family demonstrated significantly higher serum PFOS levels of the fathers than the mothers, consistent with the gender-related difference observed in the present study and other studies [3]. The higher-level similarity in the living environment and dietary between family members reduced the effect of differences in exposure, suggesting that sex-related differences in metabolism and elimination possibly play a dominant role in the body 
Table 2. Summary of serum PFOS and PFOA levels in human serum of family members from Liaoning.

\begin{tabular}{|c|c|c|c|c|c|c|c|c|c|c|}
\hline & \multirow{2}{*}{\multicolumn{2}{|c|}{ Father $(n=44)$}} & \multirow{2}{*}{\multicolumn{2}{|c|}{ Mother $(n=44)$}} & \multicolumn{6}{|c|}{ Offspring } \\
\hline & & & & & \multicolumn{2}{|c|}{ Son $(n=28)$} & \multicolumn{2}{|c|}{ Daughter $(\mathrm{n}=16)$} & \multicolumn{2}{|c|}{ Total $(n=44)$} \\
\hline & PFOS & PFOA & PFOS & PFOA & PFOS & PFOA & PFOS & PFOA & PFOS & PFOA \\
\hline Mean \pm SD & $40.1 \pm 46.1$ & $4.38 \pm 6.24$ & $23.1 \pm 23.1$ & $2.44 \pm 3.82$ & $24.6 \pm 27.8$ & $3.96 \pm 7.50$ & $20.5 \pm 29.1$ & $2.03 \pm 3.05$ & $23.1 \pm 28.0$ & $3.25 \pm 6.28$ \\
\hline GM & 20.3 & 1.88 & 12.2 & 1.13 & 9.46 & 1.49 & 7.15 & 0.98 & 8.54 & 1.28 \\
\hline Min & 0.50 & 0.28 & 0.50 & 0.18 & 0.31 & 0.21 & 0.42 & 0.31 & 0.31 & 0.21 \\
\hline $25 \%$ percentile & 9.28 & 0.53 & 5.33 & 0.40 & 3.45 & 0.40 & 2.53 & 0.40 & 2.65 & 0.40 \\
\hline Median & 26.4 & 1.85 & 14.5 & 1.20 & 12.0 & 1.75 & 6.15 & 1.05 & 11.0 & 1.65 \\
\hline $75 \%$ percentile & 55.8 & 6.85 & 39.2 & 2.98 & 48.6 & 5.05 & 37.3 & 2.38 & 40.7 & 3.60 \\
\hline $\operatorname{Max}$ & 209 & 32.9 & 108 & 16.5 & 107 & 39.8 & 106 & 12.7 & 107 & 39.8 \\
\hline
\end{tabular}

load of PFOS. It has been hypothesized that sex-related differences in metabolism, elimination, as well as exposure are attributable for the sex differences in the body load of PFOS [3]. Our previous mammalian toxicological study suggested that sex-specific routes for elimination of PFOS in females may result in relatively lower blood PFOS level than the males [20].

For PFOA, there was no significant correlation between concentrations and ages of family members. In addition, we found no correlation between paternal serum PFOS level and age. On the contrary, the results showed positive and significant associations between PFOS levels and ages of mother and offspring. Meanwhile, significant positive correlations between PFOA and PFOS concentrations were observed in father, mother and offspring.

Serum PFOS and PFOA concentrations of father, mother and offspring residing in different kinds of area are presented in Figure 2. Based upon the median PFOS and PFOA concentration, highest serum levels were observed in family members from small-medium cities. And PFOS and PFOA concentrations of family members from rural areas were almost lowest among the three groups, except that fathers residing in rural areas were higher than those in big cities. The relatively high serum levels of PFOS and PFOA in the subjects from smallmedium cities, as well as serum levels of subjects from rural areas were slightly lower than those from big cities, suggesting the prevalent human exposure to PFCs in Liaoning Province. PFOS and PFOA have been detected in the river, lakes, and tap water in Liaoning, higher than the water levels in the remote area in China [16]. Drinking water may be one of the major routes of human exposure to PFCs. Besides, other sources of exposure including the foods, atmosphere and contact with daily consumer products containing PFCs were not well known until now.

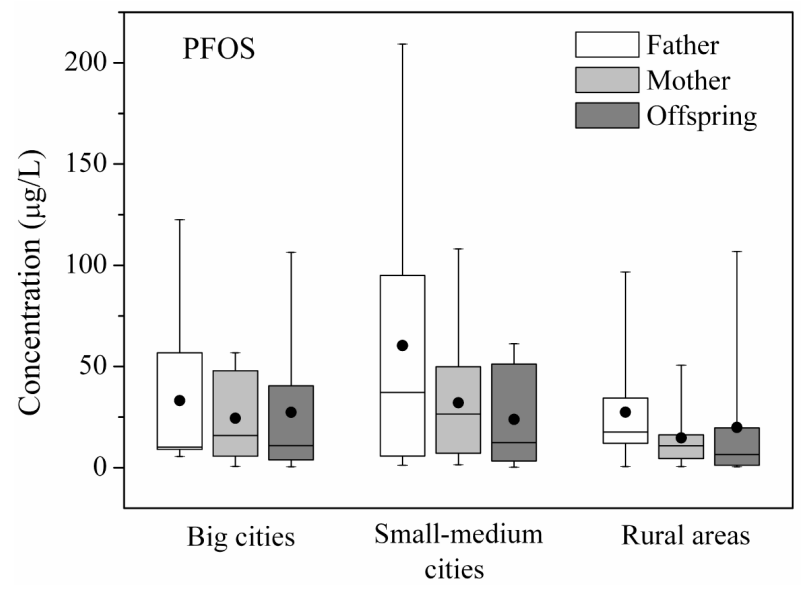

(a)

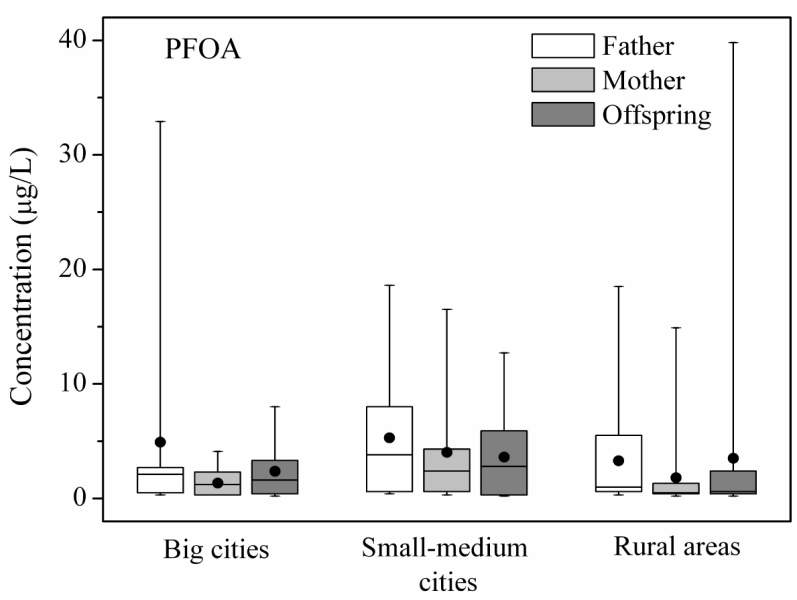

(b)

Figure 2. The serum PFOS (a) and PFOA (b) concentrations of family members from different places of residence. The box plots show the median, 25th and 75th percentiles, the lines give the 5th and 95th percentiles, while the dots present the mean values. 


\subsection{Correlation of Serum PFOS and PFOA Levels between Family Members}

Significant positive correlations were observed for serum levels of PFOS and PFOA among family members, including father and mother, father and offspring, mother and offspring, except that serum PFOS concentrations of father were not significantly correlated with those of mother, son, or daughter in the family (Table 3). Moreover, higher correlation coefficients of serum PFOS and PFOA were found between mother and offspring than that between father and offspring.

Family members have a certain period sharing similar environmental conditions, including indoor dust, contact with consumer products containing PFCs, as well as dietary intake of foods and drinking water, leading to similar exposure to PFOS and PFOA. Hence, significant correlations of exposure to PFOS and PFOA among family members were observed. However, lack of correlation of serum PFOS concentrations between father and mother, son, or daughter, suggested more unidentified factors affecting the body load of PFOS than PFOA, possibly relating to both the exposure routes and accumulation and elimination in human bodies of PFOS. Larger variability in serum PFOS levels of fathers than the mothers and offspring support the hypothesis (Figure 2). Further, the results illustrated that potential sources of PFOS might exist in some working environment and make a larger difference of exposure of the fathers with different occupation, although the subjects in the present study were recruited from those without known occupational exposure to fluorine chemicals. Therefore, exposure to PFOS from different working environment need to be further studied.

Prenatal exposure to PFCs via placental-transfer and postnatal exposure via milk constitute the background level of in offspring and even result in equal or higher PFCs concentrations in children compared with adults $[18,21]$, which possibly plays a dominant role in the strong significant correlation between serum PFCs levels of the mother and the offspring. PFOS and PFOA have been detected in cord blood and prenatal exposure and transfer of PFCs through lactation has been confirmed for humans $[14,21,22]$. Neonate exposure to PFCs in the prenatal stages has been proved to be even at similar level to the exposure at the postnatal stages [23]. PFCs have also been detected in the milk and association between increasing serum concentration and increasing milk concentrations of PFOS was observed [24]. In addition, usually longer time of the children staying with mother than father suggest more similarity in dietary and other environmental exposure, which also could contribute partly to the stronger correlation of serum PFOS and PFOA level between mother and offspring.

The associations on serum PFOA and PFOS concentrations among family members from different residential places were presented in Table 4. The results showed significant positive correlations between paternal and maternal PFOA levels in small-medium cities and rural areas. However, we only found correlation between paternal and maternal PFOS levels in rural areas. In addition, the correlation of serum concentration between father and mother from rural area was more conspicuous compared with that from big cities and small-medium cities. Moreover, paternal serum PFOA level was correlated with offspring level significantly in big cities and small-medium cities. For PFOS, significant correlation between father and offspring on serum levels was only observed in rural areas. Obviously, the data showed positive and significant correlations between maternal serum PFOA and PFOS levels and that of offspring in all three groups. Similarly, the correlations of serum PFOA and PFOS concentrations between mother and offspring from all three kinds of residence were more evident than that between father and offspring.

Although serum concentrations of PFOS and PFOA in rural areas were comparable to those in small-medium

Table 3. The associations among serum PFOA and PFOS concentrations of father, mother and offspring in Liaoning province.

\begin{tabular}{|c|c|c|c|c|c|c|c|c|c|}
\hline & & \multirow{2}{*}{\multicolumn{2}{|c|}{ Mother }} & \multicolumn{6}{|c|}{ Offspring } \\
\hline & & & & \multicolumn{2}{|c|}{ Son } & \multicolumn{2}{|c|}{ Daughter } & \multicolumn{2}{|c|}{ Total } \\
\hline & & PFOA & PFOS & PFOA & PFOS & PFOA & PFOS & PFOA & PFOS \\
\hline \multirow{2}{*}{ Father } & PFOA & $0.643^{* *}$ & & $0.533^{* *}$ & & $0.870^{* *}$ & & $0.592^{* *}$ & \\
\hline & PFOS & & 0.276 & & 0.072 & & 0.348 & & $0.396^{* *}$ \\
\hline \multirow{2}{*}{ Mother } & PFOA & & & $0.760^{* *}$ & & $0.952^{* *}$ & & $0.741^{* *}$ & \\
\hline & PFOS & & & & $0.578^{* *}$ & & $0.704^{* *}$ & & $0.734^{* *}$ \\
\hline
\end{tabular}

\footnotetext{
${ }^{* *}$ Significant correlation, $p$-value $<0.01$.
} 
Table 4. The correlation on serum PFOA and PFOS concentrations among family members from different residential places.

\begin{tabular}{|c|c|c|c|c|c|c|}
\hline & & & & Mother & & Offspring \\
\hline & & & PFOA & PFOS & PFOA & PFOS \\
\hline \multirow{4}{*}{ Big cities } & \multirow{2}{*}{ Father } & PFOA & 0.597 & & $0.683^{*}$ & \\
\hline & & PFOS & & 0.533 & & 0.132 \\
\hline & \multirow{2}{*}{ Mother } & PFOA & & & $0.951^{* *}$ & \\
\hline & & PFOS & & & & $0.635^{*}$ \\
\hline \multirow{4}{*}{ Small-mdium cities } & \multirow{2}{*}{ Father } & PFOA & $0.544^{*}$ & & $0.675^{* *}$ & \\
\hline & & PFOS & & -0.057 & & -0.043 \\
\hline & \multirow{2}{*}{ Mother } & PFOA & & & $0.890^{* *}$ & \\
\hline & & PFOS & & & & $0.624^{*}$ \\
\hline \multirow{4}{*}{ Rural areas } & \multirow{2}{*}{ Father } & PFOA & $0.686^{* *}$ & & 0.432 & \\
\hline & & PFOS & & $0.820^{* *}$ & & $0.482^{*}$ \\
\hline & \multirow{2}{*}{ Mother } & PFOA & & & $0.745^{* *}$ & \\
\hline & & PFOS & & & & $0.809^{* *}$ \\
\hline
\end{tabular}

"Significant correlation, $p<0.05 ;{ }^{* *}$ Significant correlation, $p<0.01$.

and big cities, difference in the association between family members from different regions suggested the difference in exposure pathway to some extent. Stronger correlation of serum PFOS and PFOA levels was observed among family members in rural area than those in big and small-medium cities except that between parents and offspring. Again, the larger variance in the serum PFOS and PFOA levels in big cities and small-medium cities than the rural areas suggests that extra exposure sources might exist in the outdoor or working environment in these regions.

In summary, the serum PFOS levels of residence in Xinjiang provide a reference value for human exposure assessment in China. Accordingly, the family members from Liaoning were proved to be posed to a heavier exposure than those reside in Xinjiang. The significant correlation of serum PFOS and PFOA levels among family members confirmed the importance of dietary and family indoor dust in human exposure to PFCs. Moreover, prenatal exposure to PFCs via placental-transfer and postnatal exposure via milk result in a background level in human body and were observed as a stronger correlation between mother and offspring than that between father and offspring. Further, lack of correlation of serum PFOS concentrations between father and mother, son, or daughter, as well as less correlation among family members in big and small-medium cities than in rural areas, suggested that exposure sources of PFCs in the outdoor or working environment might exist and should be further studied.

\section{Acknowledgements}

The study is financially supported by National Nature Science Foundation of China (81102096), National science \& technology pillar program during the eleventh five-year plan period (2006BAI06B02), and Program for Changjiang Scholars and Innovative Research Team in University (IRT0813)

\section{REFERENCES}

[1] M. Houde, J. W. Martin, R. J. Letcher, K. R. Solomon and D. C. G. Muir, "Biological Monitoring of Polyfluoroalkyl Substances: A Review," Environmental Science \& Technology, Vol. 40, No. 11, 2006, pp. 3463-3473. doi: $10.1021 / \mathrm{es} 052580 \mathrm{~b}$

[2] C. Lau, K. Anitole, C. Hodes, D. Lai, A. Pfahles-Hutchens and J. Seed, "Perfluoroalkyl Acids: A Review of Monitoring and Toxicological Findings," Toxicological Sciences, Vol. 99, No. 2, 2007, pp. 366-394. doi:10.1093/toxsci/kfm128

[3] H. Fromme, S. A. Tittlemier, W. Volkel, M. Wilhelm and D. Twardella, "Perfluorinated Compounds-Exposure Assessment for the General Population in Western Countries," International Journal of Hygiene and Environmental Health, Vol. 212, No. 3, 2009, pp. 239-270. doi:10.1016/j.ijheh.2008.04.007

[4] M. Murakami, E. Imamura, H. Shinohara, K. Kiri, Y. Muramatsu, A. Harada and H. Takada, "Occurrence and Sources of Perfluorinated Surfactants in Rivers in Japan," Environmental Science \& Technology, Vol. 42, No. 17, 2008, pp. 6566-6572.doi:10.1021/es800353f

[5] L. Tao, J. Ma, T. Kunisue, E. L. Libelo, S. Tanabe and K. 
Kannan, "Perfluorinated Compounds in Human Breast Milk from Several Asian Countries, and in Infant Formula and Dairy Milk from the United States," Environmental Science \& Technology, Vol. 42, No. 22, 2008, pp. 8597-8602. doi:10.1021/es801875v

[6] L. W. Y. Yeung, Y. Miyake, Y. Wang, S. Taniyasu, N. Yamashita and P. K. S. Lam, "Total Fluorine, Extractable Organic Fluorine, Perfluorooctane Sulfonate and other Related Fluorochemicals in Liver of Indo-Pacific Humpback Dolphins (Sousa chinensis) and Finless Porpoises (Neophocaena phocaenoides) from South China," Environmental Pollution, Vol. 157, No. 1, 2009, pp. 17-23. doi:10.1016/j.envpol.2008.08.005

[7] J. Holzer, O. Midasch, K. Rauchfuss, M. Kraft, R. Reupert, J. Angerer, P. Kleeschulte, N. Marschall and M. Wilhelm, "Biomonitoring of Perfluorinated Compounds in Children and Adults Exposed to PerfluorooctanoateContaminated Drinking Water," Environmental Health Perspectives, Vol. 116, No. 5, 2008, pp. 651-657. doi:10.1289/ehp.11064

[8] M. Wilhelm, J. Holzer, L. Dobler, K. Rauchfuss, O. Midasch, M. Kraft, J. Angerer and G. Wiesmuller, "Preliminary Observations on Perfluorinated Compounds in Plasma Samples (1977-2004) of Young German Adults from an Area with Perfluorooctanoate-Contaminated Drinking Water," International Journal of Hygiene and Environmental Health, Vol. 212, No. 2, 2009, pp. 142-145. doi:10.1016/i.ijheh.2008.04.008

[9] R. Vestergren and I. T. Cousins, "Tracking the Pathways of Human Exposure to Perfluorocarboxylates," Environmental Science \& Technology, Vol. 43, No. 15, 2009, pp. 5565-5575. doi:10.1021/es900228k

[10] K. Harada, N. Saito, K. Inoue, T. Yoshinaga, T. Watanabe, S. Sasaki, S. Kamiyama and A. Koizumi, "The Influence of Time, Sex and Geographic Factors on Levels of Perfluorooctane Sulfonate and Perfluorooctanoate in Human Serum over the Last 25 Years," Journal of Occupational Health, Vol. 46, No. 2, 2004, pp. 141-147. doi:10.1539/joh.46.141

[11] Y. H. Jin, N. Saito, K. H. Harada, K. Inoue and A. Koizumi, "Historical Trends in Human Serum Levels of Perfluorooctanoate and Perfluorooctane Sulfonate in Shenyang, China," Tohoku Journal of Experimental Medicine, Vol. 212, No. 1, 2007, pp. 63-70.

doi:10.1620/tjem.212.63

[12] B. H. Alexander, G. W. Olsen, J. M. Burris, J. H. Mandel and J. S. Mandel, "Mortality of Employees of a Perfluorooctanesulphonyl Fluoride Manufacturing Facility," Occupational and Environmental Medicine, Vol. 60, No. 10, 2003, pp. 722-729.

[13] B. J. Apelberg, F. R. Witter, J. B. Herbstman, A. M. Calafat, R. U. Halden, L. L. Needham and L. R. Goldman, "Cord serum Concentrations of Perfluorooctane Sulfonate (PFOS) and Perfluorooctanoate (PFOA) in Relation to Weight and Size at Birth," Environmental Health Perspectives, Vol. 115, No. 11, 2007, pp. 1670-1676. doi:10.1289/ehp.10334

[14] C. Fei, J. K. Mclaughlin, R. E. Tarone and J. Olsen, "Perfluorinated Chemicals and Fetal Growth: A Study within the Danish National Birth Cohort," Environmental Health Perspectives, Vol. 115, No. 11, 2007, pp. 1677-1682. doi:10.1289/ehp.10506

[15] N. Washino, Y. Saijo, S. Sasaki, S. Kato, S. Ban, K. Konishi, R. Ito, A. Nakata, Y. Iwasaki, K. Saito, H. Nakazawa and R. Kishi, "Correlations between Prenatal Exposure to Perfluorinated Chemicals and Reduced Fetal Growth," Environmental Health Perspect, Vol. 117, No. 4, 2009, pp. 660-667.

[16] Y. H. Jin, W. Liu, I. Sato, S. F. Nakayama, K. Sasaki, N. Saito and S. Tsuda, "PFOS and PFOA in Environmental and Tap Water in China," Chemosphere, Vol. 77, No. 5, 2009, pp. 605-611. doi:10.1016/j.chemosphere.2009.08.058

[17] Y. H. Jin, G. H. Dong, W. Q. Shu, M. Ding, C. Zhai, L. Wang, X. Liu, N. Saito and K. Sasaki, "Comparison of Perfluorooctane Sulfonate and Perfluorooctane Acid in Serum of Non-Occupational Human from Shenyang and Chongqing Areas," Journal of Hygiene Research, Vol. 35, No. 5, 2006, pp. 560-563.

[18] L. W. Y. Yeung, M. K. So, G. B. Jiang, S. Taniyasu, N. Yamashita, M. Y. Song, Y. N. Wu, J. G. Li, J. P. Giesy, K. S. Guruge and P. K. S. Lam, "Perfluorooctanesulfonate and Related Fluorochemicals in Human Blood Samples from China," Environmental Science \& Technology, Vol. 40, No. 3, 2006, pp. 715-720. doi:10.1021/es052067y

[19] K. J. Hansen, L. A. Clemen, M. E. Ellefson and H. O. Johnson, "Compound-Specific, Quantitative Characterization of Organic Fluorochemicals in Biological Matrices," Environmental Science \& Technology, Vol. 35, No. 4, 2001, pp. 766-770. doi:10.1021/es001489z

[20] W. Liu, X. Li, L. Xu, L. Liu, Y. H. Jin, I. Sata and S. Tsuda, "Influence of Gestation and Regular Bleeding on Blood Perfluorooctane Sulfonate Levels in Mice: Evaluation of Potential Factors Inducing Sex Difference in Blood Levels," Journal of Toxicological Sciences, Vol. 35, No. 3, 2010, pp. 309-306. doi:10.2131/jts.35.309

[21] H. Fromme, C. Mosch, M. Morovitz, I. Alba-Alejandre, S. Boehmer, M. Kiranoglu, F. Faber, I. Hannibal, O. GenzelBoroviczeny, B. Koletzko and W. Volkel, "Preand Postnatal Exposure to Perfluorinated Compounds (PFCs)," Environmental Science \& Technology, Vol. 44, No. 18, 2010, pp. 7123-7129. doi:10.1021/es101184f

[22] K. Inoue, F. Okada, R. Ito, S. Kato, S. Sasaki, S. Nakajima, A. Uno, Y. Saijo, F. Sata, Y. Yoshimura, R. Kishi and H. Nakazawa, "Perfluorooctane Sulfonate (PFOS) and Related Perfluorinated Compounds in Human Maternal and Cord Blood Samples: Assessment of PFOS Exposure in a Susceptible Population during Pregnancy," Environmental Health Perspectives, Vol. 112, No. 11, 2004, pp. 1204-1207. doi:10.1289/ehp.6864

[23] L. Roosens, W. D'Hollander, L. Bervoets, H. Reynders, K. Van Campenhout, C. Cornelis, R. Van Den Heuvel, G. Koppen and A. Covaci, "Brominated Flame Retardants and Perfluorinated Chemicals, Two Groups of Persistent Contaminants in Belgian Human Blood and Milk," Environmental Pollution, Vol. 158, No. 8, 2010, pp. 25462552. doi:10.1016/j.envpol.2010.05.022 
[24] A. Kärrman, I. Ericson, B. Van Bavel, P. O. Darnerud, M. Aune, A. Glynn, S. Lignell and G. Lindström, "Exposure of Perfluorinated Chemicals through Lactation: Levels of
Matched Human Milk and Serum and a Temporal Trend, 1996-2004, in Sweden," Environmental Health Perspectives, Vol. 115, No. 2, 2007, pp. 226-230. 